\title{
Editorial
}

\section{Standardization and the Practice of Medicine}

\author{
Christoph U. Lehmann, MD \\ Marlene R. Miller, MD, MSc
}

Journal of Perinatology (2004) 24, 135-136. doi:10.1038/sj.jp.7211060

If you knew — based on reliable evidence — the best way to treat a patient, you would not like to treat correctly only a fraction of your patients. However, as a group, health-care providers do just that all the time. Only a fraction of our patients may receive the treatment suggested by evidence-based trials. Treatments vary from provider to provider and vary even over time within one provider. One recent article looking only at reasons why health-care providers do not adopt simple attempts at standardization, namely clinical practice guidelines, found a myriad of "reasons" to explain this lack of providing current evidence-based care. ${ }^{1}$ Overall, health care in comparison to other high-risk industries, like aviation, suffers from a lack of standardization, and not uncommonly this oversight translates into quality and safety concerns for our patients.

Standardization affects many areas of medicine and is continuing to expand with the growth of evidence-based medicine and efforts at measuring, reporting, and improving quality. The interesting study by Porcelli ${ }^{2}$ in this issue of the Journal of Perinatology demonstrates the enormous variation that exists between different, and even within single, neonatal intensive care units in North Carolina in the ordering of parenteral nutrition. Essentially, parenteral nutrition is an intravenous medication. This being said, we have growing evidence that medication errors are a particularly susceptible area for mistakes when taking care of any pediatric population. This is due to the unavoidable need for weight-based dosing covering a wide spectrum of "typical" weights for children. ${ }^{3,4}$ The Porcelli et al. study is one of the first to document the breadth of this potential safety concern with respect to parenteral nutrition.

Overall, we have adopted and accepted standardization in many aspects of our life: it allows us to buy equipment that can be

Eudowood Neonatal Pulmonary Division and Division of Health Information Sciences (C.U.L), John Hopkins University; and Quality and Safety Initiatives Johns Hopkins Children's Center (M.R.M), Johns Hopkins University, Baltimore, MD, USA.

Address Correspondence and reprint reqsuests to Christoph U. Lebmann, MD, Department of Pediatrics, Division of Neonatology, Johns Hopkins University, 600 N. Wolfe Street, CMSC 210, Baltimore, MD 21287-3200, USA. plugged into any electric outlet or be connected to other machines and it determines our behavior in traffic. However, when attempts are made to apply more standardization to medicine, our reluctance and resistance remain high. We tend to think of standardization as a threat to the exercise of individual judgment and creativity. Our fear of "cookbook medicine" and the perceived loss of our treatment freedom are major obstacles in the implementation of standardized treatments in medicine. However, with the growth of patient safety and reporting initiatives over the last 5 years, we can no longer avoid the clear call to make sure that we consistently deliver high quality and safe care.

Standardization reduces variation in clinical treatment and patient outcome and thus improves the quality of patient care making it safer as well by reduction of potential errors. Standardization reduces waste, makes treatment predictable and controllable and defines clear responsibilities for all team members.

Clearly, standardization has its geographical and political boundaries. Standard of care may vary between different healthcare systems and countries due to a variety of financial, population-specific or ethical reasons. Nevertheless, standardization should not be limited to a single department or hospital. If experts can agree on the optimal approach, hospitals within a region or country should be able to share a standardized approach. Indeed institutions and policy makers and large health-care purchasers are clamouring for dissemination of best practices so that all patients can benefit from advances in care models. For example, the desire to standardize across local boundaries is a frequent theme in questions on the NICU-Net for information on protocols and standardized approaches to neonatal problems. ${ }^{5}$ The Internet, and information technology in general, has greatly facilitated rapid communication among providers and the ability of these providers to discuss and share standardization tactics across regions and institutions. For some types of safety concerns such as medication ordering and parenteral nutrition ordering, information technology — unlike we humans — can do the same task, without variation every time it performs the task, based on the guidelines contained in its program. Information technology is not susceptible to fatigue and mathematical errors. Based on the alarming findings in his study, Porcelli suggests the elimination of paper 
forms and the use of electronic ordering to generate parenteral nutrition orders. But why stop there? Making applications, such as a parenteral nutrition program, available through the Internet as web-based applications, ${ }^{6}$ allows institutions to standardize care without great effort. We must make the paradigm shift and stop seeing the Internet and associated information technology as just another information resource. The web has become an application platform for health-care tasks and we must focus our efforts to increase standardization and maximize safety by expanding medical web-based applications.

\section{References}

1. Cabana MD, Rand CS, Powe NR, et al. Why don't physicians follow clinical practice guidelines? A framework for improvement. JAMA 1999:282(15):1458-65.
2. Porcelli P. A survey of neonatal parenteral nutrition design practices in North Carolina. J Perinatol 2004;24:137-42.

3. Kaushal R, Bates DW, Landrigan C, et al. Medication errors and adverse drug events in pediatric inpatients. JAMA 2001;285:2114-20.

4. Vincer MJ, Murray JM, Yuill A, Allen AC, Evans JR, Stinson DA. Drug errors and incidents in a neonatal intensive care unit. Am J Dis Child 1989;143:737-40.

5. Duncan RG, Hermansen M, Hoffstatter JA, Malloy C, Lehmann CU, Henderson B. (Moderators). NICU-Net: A closed and moderated forum for discussion of neonatal intensive care issues. [8/14/2003] Online at http:// groups.yahoo.com/group/nicu-net.

6. Lehmann CU, Conner KG, Cox JM. Provider error prevention: online total parenteral nutrition calculator. Proceedings of the AMIA Symposium, 2002. pp. 435-9. 